\title{
Triboionization in Discontinuous Atmospheric Pressure Inlet for a Miniature Ion Trap Mass Spectrometer
}

Chuting $\mathrm{Xu}^{\dagger \uparrow s}$, Huiwen Ruan ${ }^{\dagger \uparrow}$, Weiguo Wang ${ }^{\dagger \S}$ and Haiyang $\mathrm{Li}^{* \dagger \S}$

${ }^{\dagger}$ Key Laboratory of Separation Science for Analytical Chemistry, Dalian Institute of Chemical Physics, Chinese Academy of Sciences (CAS), 457 Zhongshan Road, Dalian, 116023, People’s Republic of China

${ }^{\ddagger}$ University of Chinese Academy of Sciences, 19 Yuquan Road, Beijing, 100049, People’s Republic of China

${ }^{\S}$ Dalian Key Laboratory for Online Analytical Instrumentation, 457 Zhongshan Road, Dalian, 116023, People’s

Republic of China

*Corresponding Authors. Haiyang Li, email: hli@ dicp.ac.cn 


\section{Table of Contents}

1. The mass spectra of PFTBA (a) and 1,3-dichlorobenzene (b) acquired by triboionization in ITMS $\cdots . . . . . . \mathrm{S} 1$

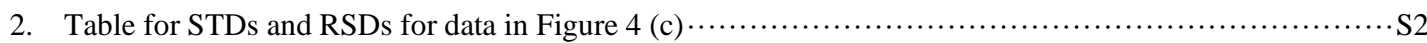

3. The observed product ions of 21 kinds of chemical compounds by triboionization method $\cdots \ldots \ldots \ldots \ldots \ldots . . . . . . . .33$ 
1. The mass spectra of PFTBA (a) and 1,3-dichlorobenzene (b) acquired by triboionization in ITMS

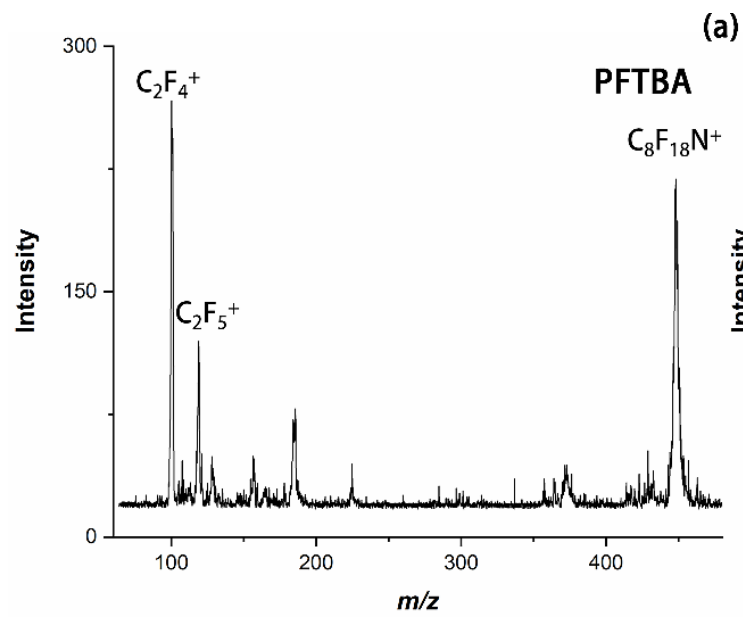

(a)

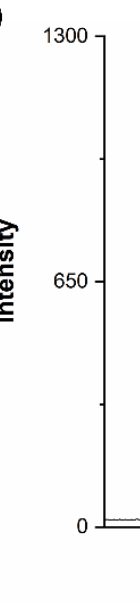

(b) 1,3-Dichlorobenzene $[\mathrm{M}]^{+}$

(M-C)enzene $[\mathrm{M}-\mathrm{Cl}]^{+}$

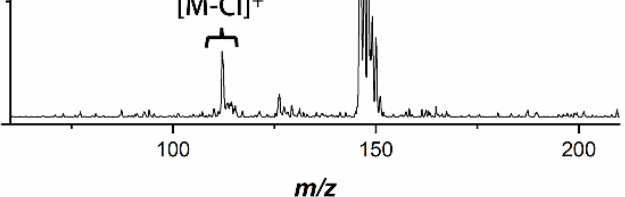

Figure S1. The mass spectra of $1 \mathrm{ppm}_{\mathrm{v}}$ PFTBA (a) and 1,3-dichlorobenzene (b) acquired by triboionization in ITMS

Figure S1 presents mass spectra of $1 \mathrm{ppm}_{\mathrm{v}}$ PFTBA (a) and 1,3-dichlorobenzene (b) acquired by triboionization in ITMS. The limits of detection (LODs) calculated based on a signal-to-noise ratio bigger than 3 were $87.5 \mathrm{ppb}_{\mathrm{v}}$ and $35.8 \mathrm{ppb}_{\mathrm{v}}$ for anisole and DMMP respectively.

\section{Table for STDs and RSDs for data in Figure 4 (c)}

Table S1. STDs and RSDs for data in Figure 4 (c)

\begin{tabular}{|c|c|c|c|c|c|c|}
\hline $\begin{array}{c}\text { Time difference } \\
\text { between valves } \\
\text { opening/ms }\end{array}$ & 8 & 7 & 6 & 4 & 2 & 0 \\
\hline $\mathrm{AVG}$ & 41.4 & 2295.8 & 6067.6 & 6811.2 & 6732.4 & 6571.1 \\
\hline $\mathrm{STD}$ & 14.8 & 1262.7 & 493.5 & 1241.4 & 856.2 & 1196.6 \\
\hline $\mathrm{RSD}$ & 35.7 & 55.0 & 8.1 & 18.2 & 12.7 & 18.2 \\
\hline
\end{tabular}

Figure 4 (c) shows the ionization efficiency trend as a function of pressure change during triboionization process. The standard deviation (STDs) and relative standard deviation (RSDs) are listed in Table S1. 
3. The observed product ions of 21 kinds of chemical compounds by triboionization method

Table S1. The observed product ions of 21 kinds of chemical compounds by triboionization method.

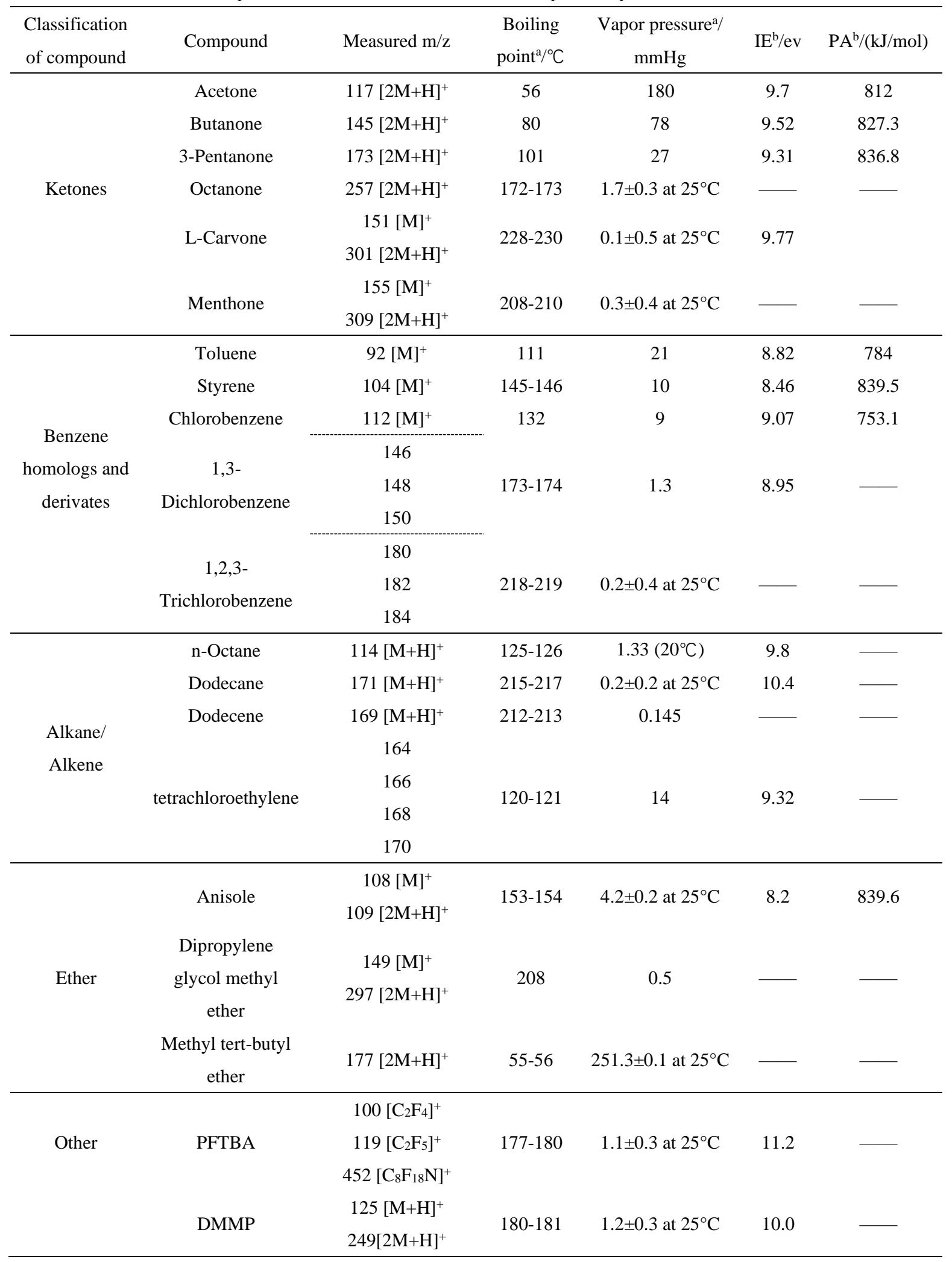

1. ${ }^{\mathrm{a} D a t a}$ are obtained from ChemSpider. ${ }^{\mathrm{b}}$ Data are obtained from NIST.

2. Abbreviations: PFTBA: perfluorotributylamine; DMMP: dimethyl methylphosphonate.

Table S1 shows the physical and chemical parameters and product ions of 21 kinds of chemical compounds by triboionizaiton. 